\title{
Linear Instability of an Anticyclonic Vortex in a Two-Layer Ocean
}

\author{
Nathan Paldor ${ }^{1}$ and Doron NoF \\ Department of Oceanography and Geophysical Fluid Dynamics Institute, Florida State University, Tallahassee
}

\begin{abstract}
The linear stability of a (zero potential vorticity) circular lens (i.e., warm-core ring) is investigated for the case where the ocean surrounding the lens is of finite depth. The hydrostatic balance across the lens' interface couples the pressure variations inside the lens with those in the surrounding ocean which produces the instability. It is found that in contrast to lenses in an infinitely deep ocean which are always stable, lenses in a finite-depth ocean are unstable. For a fixed lens thickness the growth rates (instability exponents) increase with decreasing total depth. For each azimuthal wave number there exists a total (ocean) depth cutoff value above which the lens is stable and below which the lens is unstable; this cutoff value increases with wave number. In most cases, instability would occur whenever the ocean-lens depth ratio is less than 3 or 4 , suggesting that actual rings in the ocean may become unstable as they approach the shore where the ocean depth is relatively small.
\end{abstract}

\section{INTRODUCTION}

The addition of an active second layer to an otherwise stable ocean has been recently shown to induce vigorous instabilities along geostrophic fronts [Griffiths et al., 1982; Killworth et al., 1984; Paldor and Ghil, 1990, also Shortwave instabilities of coastal currents: Application to the Algerian Current, submitted to Journal of Physical Oceanography, 1990]. These instabilities owe their existence to the outcropping of isopycnal surfaces; the shortwave growth rates associated with them are of the order of 1 day. Since the real ocean has a finite depth, it is of interest to investigate the stability of a circular lens when the dynamics of the surrounding ocean is taken into account.

\subsection{Background}

The stability of a circular lens containing whirling light water overriding an infinitely deep (hence passive) ocean has been studied by Killworth [1983], who showed that when the shape of the interface separating the lens from the ocean is parabolic, the flow within the lens is stable so that no growing perturbations exist. The inverted case where a lens of heavy water is underlying an infinitely thick ocean above it has, of course, the same stability properties. Other eddy stability studies were done by Griffiths and Linden [1981], Cushman-Roisin [1986], Ripa [1987], and Flierl [1988], but these studies do not deal directly with the problem at hand where a lens is embedded in a finite-depth ocean which is initially at rest (Figure 1). The studies that are most closely related to our problem are those of Helfrich and Send [1988] and Ripa [1989]. Helfrich and Send [1988] studied the stability of a quasi-geostrophic two-layer ocean with a circular vortex of constant potential vorticity in each layer. Their study clearly demonstrates the effect of the second layer in the generation of instabilities. The growth rates in this case increase with a decreasing depth ratio (ratio of the upper layers to the lower layer depth). Ripa [1989] derived

\footnotetext{
${ }^{1}$ Permanently at Institute of Earth Sciences, Hebrew University of Jerusalem, Jerusalem, Israel.

Copyright 1990 by the American Geophysical Union.

Paper number 90JC01540.

0148-0227/90/90JC-01540\$05.00
}

integrated constraints for stratified eddies whose potential vorticity is not uniform.

\subsection{Present Approach}

To simplify the algebraic expressions, we assume that the potential vorticity of the flow within the lens is zero. This assumption is based on earlier studies which show that the stability of a given flow was not drastically altered when the potential vorticity was changed [Paldor and Killworth, 1987]. This is especially true in cases such as a lens where the absolute vorticity has to vanish along the edge as its height vanishes there. With the potential vorticity being equal to zero everywhere within the lens, the shear of the azimuthal velocity must (on an $f$ plane) be constant so that the velocity itself increases linearly with the radius of the lens and the interface depth is parabolic. We shall show that vigorous instabilities occur in this case and that for any given value of the depth ratio there might exist a few instability exponents differing from each other mainly by the value of the azimuthal wave number.

Lenses with uniform potential vorticity were first studied theoretically by Csanady [1979], who neglected the centrifugal terms in the equations of motion and derived a simple estimate of the shape and frictional lifetime. Flierl [1979] added the centrifugal terms to the equations of motion and showed that these can never be neglected in the lens' dynamics. Both studies showed that in general, there are no analytical solutions for lenses embedded in a resting ocean unless they have zero potential vorticity. It is for this reason that we chose to focus our attention on basic states where the potential vorticity vanishes.

\section{Formulation}

Consider again the lens displayed in Figure 1; the ocean is of finite depth and rotates about the vertical with a frequency $f / 2$. The mean flow outside the lens is assumed to be zero so the pressure there is constant; as mentioned, the mean flow within the lens is assumed to have zero potential voriticity.

\subsection{Governing Equations and Boundary Conditions}

The nondimensionalization is carried out by scaling time on $1 / f$ (where $f$ is the Coriolis parameter), lengths on $L$ (the 


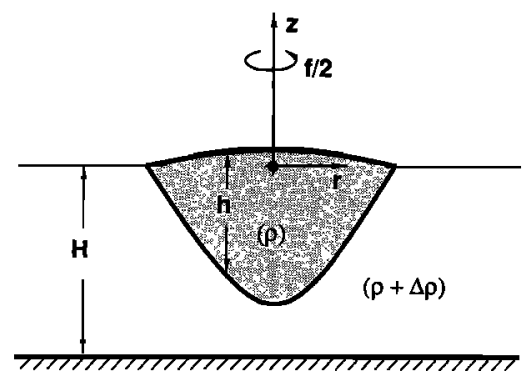

Fig. 1. Schematic diagram of the lens under study. From a mathematical point of view the light lens is analogous to a heavy lens on the bottom of a finite-depth ocean.

unperturbed radius of the lens), velocities on $L f$, pressures on $L^{2} f^{2} / \rho$, and heights (depths) on $L^{2} f^{2} / g^{\prime}$ (where $g^{\prime}=$ $g\left(\rho_{2}-\rho_{1}\right) / \rho_{2}$ is the reduced gravity). The radial $(r)$ and azimuthal $(\theta)$ momentum equations for the velocity components in these directions ( $u$ and $v$, respectively) and the continuity equation within the lens take the nondimensional form

$$
\begin{gathered}
\frac{\partial u}{\partial t}+u \frac{\partial u}{\partial r}+\frac{v}{r} \frac{\partial u}{\partial \theta}-\frac{v^{2}}{r}-v+\frac{\partial p}{\partial r}=0 \\
\frac{\partial v}{\partial t}+u \frac{\partial v}{\partial r}+\frac{v}{r} \frac{\partial v}{\partial \theta}+\frac{u v}{r}+u+\frac{1}{r} \frac{\partial p}{\partial \theta}=0 \\
\frac{\partial h}{\partial t}+\frac{1}{r} \frac{\partial}{\partial r}(r u h)+\frac{1}{r} \frac{\partial}{\partial \theta}(v h)=0
\end{gathered}
$$

where $p$ is the pressure within the lens and $h$ is its thickness (which vanishes, of course, for $r \geq 1$ ).

The assumption of zero potential vorticity in the lens yields

$$
1+\frac{\partial v}{\partial r}+\frac{v}{r}-\frac{\partial u}{\partial \theta}=0
$$

which is not an independent equation, but rather can be used to replace one of the momentum equations (1) or (2).

In the surrounding ocean the $r$ and $\theta$ momentum equations and the continuity equation are

$$
\begin{gathered}
\frac{\partial u}{\partial t}+u \frac{\partial u}{\partial r}+\frac{v}{r} \frac{\partial u}{\partial \theta}-\frac{v^{2}}{r}-v+\frac{\partial \Phi}{\partial r}=0 \\
\frac{\partial v}{\partial t}+u \frac{\partial v}{\partial r}+\frac{v}{r} \frac{\partial v}{\partial \theta}-\frac{u v}{r}+u+\frac{1}{r} \frac{\partial \Phi}{\partial \theta}=0 \\
\frac{\partial(H-h)}{\partial t}+\frac{1}{r} \frac{\partial}{\partial r}[r u(H-h)]+\frac{1}{r} \frac{\partial}{\partial \theta}[v(H-h)]=0
\end{gathered}
$$

where $\Phi$ is the pressure outside the lens and $H$ is the total (nondimensional) depth of the ocean.

The hydrostatic balance across the lens' interface implies

$$
p=\Phi+h
$$

The above equations are subject to the following boundary conditions. First, within the surrounding water the velocity and pressure fluctuations must vanish away from the lens.
Second, the lens depth must vanish along a curve whose position and shape must be determined as a part of the problem. Third, both the velocity and the pressure must be well behaved at the center $(r=0)$ and circumference $(h=0)$.

\subsection{The Basic State}

As briefly mentioned, we shall take a mean state where (1) the flow outside the lens vanishes (so the pressure can be assumed zero everywhere there) and (2) the steady flow within the lens is purely azimuthal. From (4) we conclude that the mean azimuthal velocity $v_{0}$ satisfies

$$
\frac{\partial v_{0}}{\partial r}+\frac{v_{0}}{r}+1=0
$$

which has the solution (which is regular at $r=0$ )

$$
v_{0}=-r / 2
$$

Note that this velocity profile corresponds to the relative vorticity that counterbalances the planetary vorticity.

For this mean flow the hydrostatic balance, (8), implies that $p=h$ so that the radial momentum equation in the lens, (5) (with $u=0$ ), implies that the mean depth of the lens' interface, $h_{0}$, satisfies the equation

$$
\partial h_{0} / \partial r=v_{0}\left(1+v_{0} / r\right)
$$

which, for the velocity given by (10), gives

$$
h_{0}=\left(1-r^{2}\right) / 8
$$

In what follows we shall assume that $H>\frac{1}{8}$, which, according to our scaling, assures that the maximum lens depth is smaller than the total ocean depth.

\section{Perturbation Analysis}

\subsection{Perturbed Equations}

We now proceed in the usual way by linearizing the governing equations about the mean state and assuming that the perturbations (i.e., small-amplitude deviations from this mean state) have an azimuthal wave structure:

$$
\exp [i n(\theta-c t)]
$$

where $n$ is the azimuthal wave number (taken to be an integer on account of the $\theta \rightarrow \theta+2 \pi$ symmetry) and $c$ is the phase speed so that $w(=n c)$ is the frequency of the wave.

Instabilities are associated with a complex value of $c=$ $c_{r}+i c_{i}$ (where $i=\sqrt{-1}$ ), at which case the wave's temporal behavior is dominated by an exponential growth at a rate exp $\left(n c_{i} t\right)$. Note that the exponentially decaying solution exp $\left(-n c_{i} t\right)$ exists, of course, too but is of lesser interest.

After some straightforward (but tedious) eliminations the linearized lens equations (1)-(3) become

$$
\frac{\partial}{\partial r}\left(r h_{0} \frac{\partial p}{\partial r}\right)-\left(n^{2} h_{0} / r\right) p+r n^{2}\left(c+\frac{1}{2}\right)^{2} h=0
$$

A similar procedure is now applied to the surrounding ocean, and this yields

$$
\begin{array}{r}
\frac{\partial}{\partial r}\left[r\left(H-h_{0}\right) \frac{\partial \Phi}{\partial r}\right]-\left[n^{2}\left(H-h_{0}\right) / r-\frac{\partial h_{0}}{\partial r} / c\right] \Phi \\
+r\left(1-n^{2} c^{2}\right) h=0
\end{array}
$$


We proceed by using (8) to eliminate $h$ from (13) and (14). This gives the following coupled equations for the pressure perturbations inside and outside the lens:

$$
\frac{\partial}{\partial r}\left(r h_{0} \frac{\partial p}{\partial r}\right)-\left[n^{2} h_{0} / r-r n^{2}\left(c+\frac{1}{2}\right)^{2}\right] p-\left[r n^{2}\left(c+\frac{1}{2}\right)^{2}\right] \Phi=0
$$$$
\frac{\partial}{\partial r}\left[r\left(H-h_{0}\right) \frac{\partial \Phi}{\partial r}\right]-\left[n^{2}\left(H-h_{0}\right) / r-\frac{1}{c} \frac{\partial h_{0}}{\partial r}+r\left(1-n^{2} c^{2}\right)\right] \Phi
$$$$
+r\left(1-n^{2} c^{2}\right) p=0
$$

These equations are subject to the boundary conditions which are discussed below.

\subsection{Derivation of the Boundary Conditions}

Outside the lens $(r \geq 1)$ the pressure equation (14) reduces to

$$
r \frac{\partial}{\partial r}\left(r \frac{\partial \Phi}{\partial r}\right)-n^{2} \Phi=0
$$

and its regular (i.e., decaying at infinity) solution is $\Phi=$ $A r^{-n}$, where $A$ is a constant to be determined. Along the edge ( $r=1)$, both $\Phi$ and its derivative must be matched to the solution directly underneath the lens $(r<1)$ so that the constant $\boldsymbol{A}$ can be easily determined. Taking this into account, we arrive at the following boundary condition:

$$
\frac{\partial \Phi}{\partial r}(1)+n \Phi(1)=0
$$

which assures the continuity of both $\Phi$ and $\partial \Phi / \partial r$.

As for the pressure inside the lens $p$, we note that its differential equation (15) is singular at $r=1$ (where $h_{0}$ vanishes) so that singular solutions exist there. Expanding (15) about the singular point $r=1$ and requiring that the solution be regular there, we get the boundary condition for p,

$$
\frac{\partial p}{\partial r}(1)=4 n^{2}\left(c+\frac{1}{2}\right)^{2}[p(1)-\Phi(1)]
$$

The point $r=0$ is singular for both (15) and (16), and the regularity requirement there yields the solution at the center,

$$
\begin{aligned}
& p \rightarrow r^{n} \\
& \Phi \rightarrow r^{n} \\
& r \rightarrow 0
\end{aligned}
$$

The coupled set (15) and (16) (with $v_{0}$ and $h_{0}$ given by (10) and (12)) along with the four boundary conditions (17)-(19) is solved numerically in the next section, where the eigenvalues $c$ are found as a fucntion of $\boldsymbol{H}$ for various values of $\boldsymbol{n}$.

\section{Solution AND INSTABILITY EXPONENTS}

The numerical solution of (14)-(16) subject to (17)-(19) is not at all trivial, and for its details the reader is referred to Paldor and Killworth [1987] and Paldor and Ghil [1990], who deal with very similar cases. The essence of the technique is to integrate the equations away from the endpoints $(r=0,1)$ toward the middle of the ring $\left(r=\frac{1}{2}\right)$, where the numerical values of $\Phi, p$, and their derivatives are matched. These matching conditions then give the appropriate values of $\Phi$ and $p($ at $r=0,1)$ and the value of $c$.

Specifically, the numerical search for the eigensolution (eigenfunctions $p$ and $\Phi$ and eigenvalue $c$ ) begins by carrying out two trial integrations from the point $r=0$ to the midpoint $r=\frac{1}{2}$. The first integration is executed with $p(0)=0$ and $\Phi(0)=1$, and the second integration with $p(0)=1$ and $\Phi(0)=0$. The four values of $p$ and $\Phi$ and their derivatives at $r=\frac{1}{2}$ resulting from these two runs are temporarily stored. Two more integrations are then carried out from the other singular point, $r=1$, to the midpoint $r=\frac{1}{2}$, and the corresponding values are also stored. For all of $p, \partial p / \partial r, \Phi$, and $\partial \Phi / \partial r$ to be continuous across $r=\frac{1}{2}$ the determinant of the $4 \times 4$ (complex) matrix (composed of the values of $p$, $\partial p / \partial r, \Phi$, and $\partial \Phi / \partial r$ at $r=\frac{1}{2}$ from the four trial runs) has to vanish. Hence by varying the eigenvalue $c$, for a given $l$ and for a fixed value of $\boldsymbol{H}$ we can find those values of $\boldsymbol{c}$ for which the determinant does indeed vanish. There are, of course, infinitely many real eigenvalues, $c=c_{r}$, representing stable waves, but these are of no interest here.

The results of this numerical search are shown in Figure 2 for several values of $n$ as a function of the depth ratio $R$, which is defined as the ratio of the total depth to the maximum lens depth (i.e., $R=8 H$ ). No instabilities were found for the $n=1$ mode or for even modes with $n>2$. The eigenfunctions have the classical boundary layer structure with vanishing amplitudes at $r=0$ (both $P$ and $\Phi$ ) and $r \rightarrow \infty$ (that of $\Phi$ ) so that the maximal amplitudes are encountered along the lens' edge, $\rightarrow=1$.

\section{Summary AND Discussion}

We have shown in this study that an active surrounding ocean has a destabilizing effect on a circular lens of zero potential vorticity. This is to be contrasted with the single active layer case which is known to be stable [e.g., Killworth, 1983; Flierl, 1984]. The general tendency of the instability exponents is to increase with decreasing total ocean depth (for a fixed lens thickness) and to increase for lower azimuthal wave numbers. These tendencies were also found in the quasi-geostrophic, two-layer vortex of Helfrich and Send [1988]. It is also consistent with the numerical experiments of Chassignet and Nof [1989] where the $\beta$-induced propagation of a lens in a finite-depth ocean was examined. In these experiments it has been noted that as the bottom was gradually brought up from infinity, the lenses became less and less stable until they ultimately broke up (when the lens depth was of the same order as the surrounding ocean). Similar results were obtained by Tang [1989] in his $f$ plane and $\beta$ plane runs.

Note that neither instabilities of even modes larger than 2 nor instabilities corresponding to $n=1$ were found despite extensive searches. It is also worth pointing out that the real part of the phase speed, $c_{r}$, is negative in all instabilities so that a "critical layer" (where $v_{0}-c_{r}$ vanishes inside the lens) is formed. We note, however, that at this layer the equations remain regular because the coefficients of the highest-order derivatives do not vanish there.

The energetics of the perturbations can be investigated using the usual technique [e.g., Killworth et al., 1984]. Namely, the linearized radial and tangential momentum 


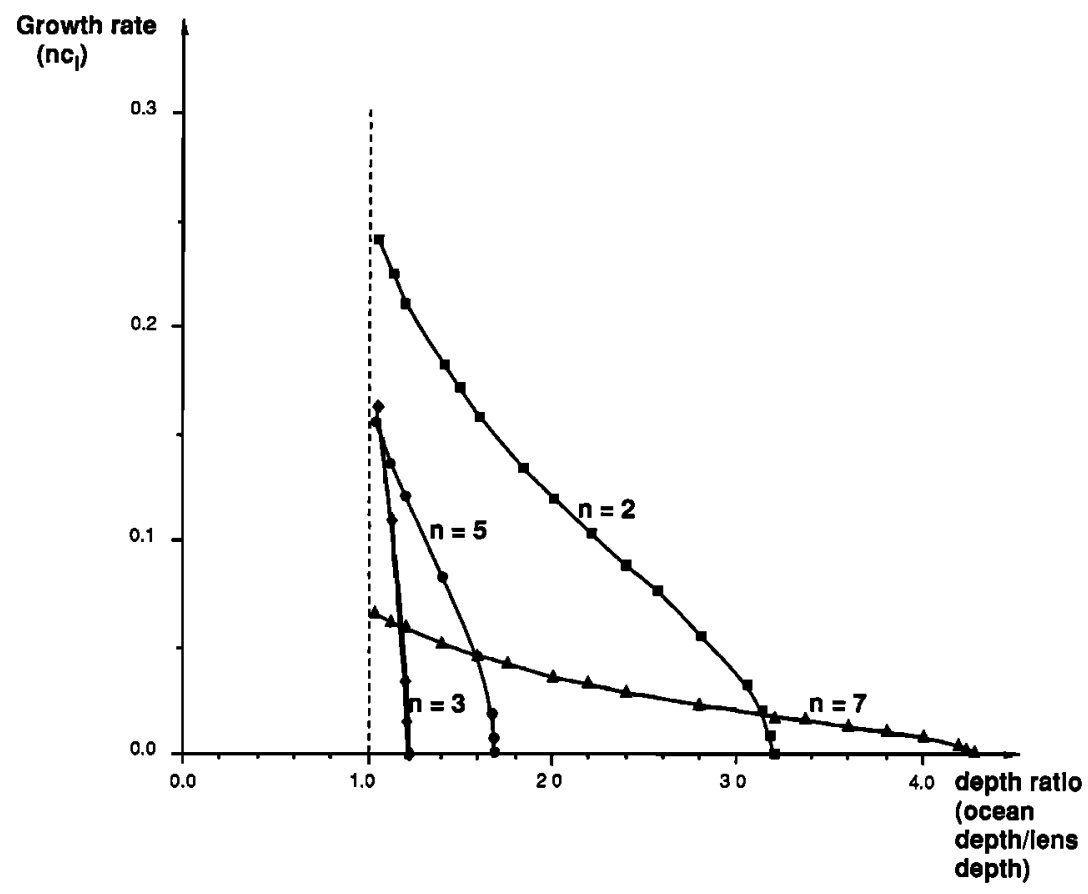

Fig. 2. The perturbation growth rates (for various wave numbers $(n))$ as a function of the depth ratio $(\cong 8 H)$. The dashed line corresponds to a lens that "kisses" the bottom, and a large ratio corresponds to a very deep surrrounding ocean. The triangles, circles, diamonds, and squares denote the numerically computed values. Note that the growth rate increases with decreasing total depth.

equations (in each of the layers) are multiplied by the mean height of the layer $\left(h_{0}\right.$ for the lens, $\left(H-h_{0}\right)$ for the lower layer) and the corresponding perturbation velocity, and the lens continuity equation is multiplied by the perturbed height. Averaging the equations with respect to $\theta$ and rearranging, we get the following energy equation:

$$
\begin{aligned}
\frac{\partial E}{\partial t}+h_{0} \frac{\partial v_{0}}{\partial r}\langle & \left.u_{1} \frac{\partial v_{1}}{\partial \theta}\right\rangle+v_{0}\left\langle\Phi \frac{\partial h}{\partial \theta}\right\rangle \\
& +\frac{\partial}{\partial r}\left\langle h_{0} u_{1}(\Phi+h)+\left(H-h_{0}\right) u_{2} \Phi\right\rangle=0
\end{aligned}
$$

where

$$
E \equiv\left\langle h_{0}\left(\frac{u_{1}^{2}}{2}+\frac{v_{1}^{2}}{2}\right)+\frac{h^{2}}{2}+\left(H-h_{0}\right)\left(\frac{u_{2}^{2}}{2}+\frac{v_{2}^{2}}{2}\right)\right\rangle
$$

and

$$
\langle\rangle \equiv \frac{r}{2 \pi} \int_{0}^{2 \pi} d \theta
$$

Here the subscripts 1 and 2 denote the lens and the surrounding layer, respectively.

Equation (20) is not integrated from $r=0$ to $r \rightarrow \infty$; the last term vanishes because \langle\rangle$=0$ at $r=0$ and all eigenfunctions tend to zero as $r \rightarrow \infty$ (see section 3.2). The result is

$\frac{\partial}{\partial t} \int_{0}^{\infty}\langle E\rangle d r+\int_{0}^{\infty} h_{0} \frac{\partial v_{0}}{\partial r}\left(u_{1} \frac{\partial v_{1}}{\partial \theta}\right) d r$

$$
+\int_{0}^{\infty} v_{0}\left(\Phi \frac{\partial h}{\partial \theta}\right) d r=0
$$

which shows that the source of energy for the perturbations' growth is mixed barotropic (varies as $h_{0} \partial v_{0} / \partial r$ ) and baroclinic $\left(v_{0}\right)$ instability. The barotropic term is affected by the Reynolds stress $\left\langle u_{1} \partial v_{1} / \partial \theta\right\rangle$, while the baroclinic term depends on the correlation of the pressures in the two layers, $\langle\Phi \partial h / \partial \theta\rangle=\langle\Phi \partial P / \partial \theta\rangle$.

The implications of this theory to the lifetime of oceanic lenses is that as long as the lens is embedded in a very deep ocean it will maintain its shape and azimuthal velocity for very long times. (By "very deep" we mean that the ocean is at least $\mathbf{4 2}$ times larger than the lens depth.) Only in cases when the surrounding ocean is relatively shallow (i.e., twice or 3 times the lens' thickness) will the instabilities found in this study be active. This may explain why the warm-core rings north of the Gulf Stream (which are embedded in an ocean 5 times deeper than their own maximum depth) maintain their shape for many months. Furthermore, it is consistent with the observations of Brenner et al. [1990], who identified an anticyclonic eddy which persisted for at least 2 years. The eddy was embedded in a layer about 5 times deeper than its maximum depth, suggesting that it was stable to small perturbations.

Two additional comments should be made with regard to our analysis. First, note that the large wave number instabilities shown in Figure 2 are probably difficult to excite; also, there might be nonlinear limitations on the final value of their amplitude. Second, our typical perturbation $e$-folding 
time is a few days which corresponds to a relatively fast growth rate.

Finally, it would be desirable to extend this study to finite (nonzero) potential vorticities. However, since there is no analytical solution for such a basic state, perturbation equations (analogous to (15)-(16)) with explicit expressions for the coefficients (see, for example, (10) and (12)) cannot be derived, which makes the numerical search for the eigensolutions very difficult.

Acknowledgments. This study was supported by National Science Foundation grant OCE-8711030 and Office of Naval Research grant N00014-89-J-1606. The skillful aid we received from Beth Raynor and Steve Van Gorder in typing the manuscript and in producing the graphs is greatly appreciated. N.P. thanks the Department of Oceanography and the Geophysical Fluid Dynamics Institute at FSU for a very enjoyable visit during which this work was initiated. Contribution 298 of the Geophysical Fluid Dynamics Institute, Florida State University.

\section{REFERENCES}

Brenner, S., Z. Rozentraub, J. Bishop, and M. Krom, The mixed layer/thermocline cycle of a persistent warm core eddy in the eastern Mediterranean, Dyn. Atmos. Oceans, in press, 1990.

Chassignet, E., and D. Nof, The propagation of isolated nonlinear eddies in a finite depth ocean, Tech. Rep. 1-90, 15 pp., Fla. State Univ., Tallahasssee, 1989.

Csanady, G. T., The birth and death of a warm core ring, $J$. Geophys. Res., 84, 777-780, 1979.

Cushman-Roisin, B., Linear stability of large, elliptical warm-core rings, J. Phys. Oceanogr., 16, 1158-1164, 1986.

Flierl, G. R., A simple model for the structure of warm and cold core rings, J. Geophys. Res., 84, 781-786, 1979.
Flierl, G. R., Rossby wave radiation from a strongly nonlinear warm eddy, J. Phys. Oceanogr., 14, 47-58, 1984.

Flierl, G. R., On the instability of geostrophic vortices, J. Fluid Mech., 197, 349-388, 1988.

Griffiths, R. W., and P. F. Linden, The stability of vortices in a rotating, stratified fluid, J. Fluid Mech., 105, 283-316, 1981.

Griffiths, R. W., P. Killworth, and M. Stern, Ageostrophic instability of ocean currents, $J$. Fluid Mech., II7, 343-377, 1982.

Helfrich, K. R., and U. Send, Finite-amplitude evolution of twolayer geostrophic vortices, J. Fluid Mech., 197, 331-348, 1988.

Killworth, P. D., On the motion of isolated lenses on a beta-plane, J. Phys. Oceanogr., 13, 368-376, 1983.

Killworth, P. D., N. Paldor, and M. E. Stern, Wave propogation and growth on a surface front in a two-layer geostrophic current, $J$. Mar. Res., 42, 761-785, 1984.

Paldor, N., and M. Ghil, Finite-wavelength instabilities of a coupled density front, J. Phys. Oceanogr., 20, 114-123, 1990.

Paldor, N., and P. Killworth, Instabilities of a two-layer coupled front, Deep Sea Res., 34, 1525-1539, 1987.

Ripa, P., On the stability of elliptical vortex solutions of the shallow-water equations, $J$. Fluid Mech., 183, 343-363, 1987.

Ripa, P., On the stability of ocean vortices, in Mesoscale/Synoptic Coherent Structures in Geophysical Turbulence, Elsevier Oceanogr. Ser., vol. 50, edited by J. Nihoul and B. Jamart, pp. 167-180, Elsevier, New York, 1989.

Tang, B., Numerical modeling of geostrophic turbulence and eddy evolution using generalized geostrophic equations, Ph.D. disser.tation, 72 pp., Fla. State Univ., Tallahassee, 1989.

D. Nof, Department of Oceanography, Florida State University, Tallahassee, FL 32306.

N. Paldor, Institute of Earth Sciences, Hebrew University of Jerusalem, Jerusalem, 91904 Israel.

(Received April 6, 1990; revised June 11, 1990; accepted June 13, 1990.) 